\title{
Temporal Pattern of Circulating Antigens and Antibody Responses in Cats Experimentally Infected with Dirofilaria immitis
}

Kris Curtis', Tom O'Connor', Leif Lorentzen', John McCall², Michael Tillson³, Ray Dillon ${ }^{3}$, Ramaswamy Chandrashekar ${ }^{1} \triangle$

${ }^{1}$ IDEXX Laboratories, Inc., Westbrook, ME 04092, USA

2 University of Georgia, Athens, GA 30602, USA

3 Auburn University, Auburn, AL 36849, USA

\section{Corresponding author:}

\section{Ramaswamy Chandrashekar}

$\triangle$ E-mail: Chandra@idexx.com

\section{Abstract}

Heartworm disease in cats has been attributed to immature adult heartworms reaching the pulmonary arteries approximately 2.5-4 months post infection and the presence of mature adult worms in the cardiopulmonary system approximately 6 months after infection. The arrival and death of immature heartworms causes significant lung pathology, a condition referred to as heartwormassociated respiratory disease (HARD). To evaluate the humoral immune response to Dirofilaria immitis, 12 cats were each infected by subcutaneous injection of 100 third-stage larvae. Postinfection serum samples were collected weekly for 4 weeks, every other week for the following 4 weeks, then monthly to day 270 for evaluation of antibodies to $D$. immitis recombinant antigens
HWAg-1 and HWAg-2 and circulating heartworm antigen (HWAg) using the PetChek ${ }^{\circledR}$ HTWM PF Test Kit (IDEXX Laboratories). Necropsies were performed 278-299 days post infection for collection of adult worms. Eleven cats were HWAg1 antibody-positive 68 days post-infection, and the remaining cat became positive by day 140 . Eleven cats were positive for HWAg-2 antibody 42-84 days post infection; all 11 remained HWAg-2 antibodypositive through day 270. Circulating HWAg was detected in 10 cats, 2 by day 140 and 8 others by day 168. The 2 antigen-negative cats had no adult worms at necropsy. This study demonstrates that decomposition of immature adult heartworms can result in detectible levels of circulating antigen prior to sexual maturation. In this experimental 
model, $D$. immitis antigen was detectable in cats at time points (days 140 and 168) associated with HARD from dying immature adult heartworms.

\section{Introduction}

Cats infected with heartworm (Dirofilaria immitis) can be asymptomatic or present most commonly with respiratory and/or gastrointestinal symptoms (Dillon 1984, Atkins et al. 2000). Cats that develop adult heartworm infection can suffer significant complications, including coughing, dyspnea, vomiting, and sudden death (McCall et al. 1992, Lister et al. 2008, Blagburn and Dillon 1984, Dillon et al. 2014). Immature adult heartworms reaching the pulmonary arteries at approximately 2.5-4.0 months post infection can cause significant lung pathology in infected cats, a condition referred to as heartworm-associated respiratory disease or HARD (Dillon et al. 2007). This stage is associated with an intense immune response, which results in the elimination of most of the immature worms and occurs regardless of whether adult worms are present or will develop at some future time (Dillon et al. 2008, Lee et al. 2010, Maia et al. 2011).

Heartworm infection can be difficult to diagnose in cats with naturally acquired infections (Berdoulay et al. 2004). Currently available heartworm antibody tests can detect feline infection as early as 1 to 2 months after infection, and are useful as the infection develops in cats that may be antigennegative but have heartworms that have developed to at least the immature adult L4 stage (Lee et al. 2010). However, antibody test sensitivity is unpredictable in cats with early as well as late infections (Snyder et al. 2000) and may be positive in cats that are placed on preventative medications after initiation of the infection (Dillon et al. 2013).

The antigen (HWAg) in detection assays is present on numerous $D$. immitis proteins, with concentra- tions adequate to be detectable in circulation as they are primarily shed by mature adult-stage female worms (Weil et al. 1985, Weil 1987). Circulating antigen can be difficult to detect due, in part, to the fact that cats usually have worms that are small and few (1-3 worms), which limits the amount of available circulating antigen and can result in all-male worm infections (Jones et al. 2014).

Reliable tests are needed for accurately detecting both early- and late-stage heartworm infection in cats. The purpose of this study was to use currently available diagnostic methods to document temporal changes in heartworm-specific antibody and HWAg in experimentally infected cats to better characterize testing options.

\section{Materials and methods}

\section{Animals and samples}

Twelve domestic shorthair, specific pathogen-free male cats (4 months old) were acclimated for 7 days before this 9-month study. Cats were injected subcutaneously with 100 infective, third-stage larvae $\left(\mathrm{L}_{3}\right)$ of $D$. immitis (day 0). Serum samples were collected from each cat at days $-1,7,14,21,28,42,56$, $68,75,84,112,140,168,189,217,245$, and 270 . All serum samples were frozen at $-80^{\circ} \mathrm{C}$ immediately after collection. Cat AOW4 died from lung disease day 140 post infection (p.i.) (Table 1 ). The remaining 11 cats were euthanized days 260-290 p.i. with an intraperitoneal overdose of pentobarbital. At the time of euthanasia, serum samples were collected directly from the right ventricle of the heart by catheterization. At necropsy, the heart, lungs, and associated vessels were removed and dissected for recovery of adult heartworms and worm fragments. The heartworms were evaluated for viability in Hanks solution at $32^{\circ} \mathrm{C}$ and counted by sex. 
Table 1 Adult heartworm and fragment counts determined in cats infected by SC injection of 100 third-stage Dirofilaria immitis larvae and necropsied 278-299 days post infection

\begin{tabular}{|c|c|c|c|}
\hline \multirow{2}{*}{ Cat ID } & \multicolumn{3}{|c|}{ Viable Heartworms } \\
\hline & Male & Female & Fragments \\
\hline AOS1 & 0 & 0 & 0 \\
\hline AOS2 & 6 & 2 & 0 \\
\hline AOU1 & 0 & 0 & 0 \\
\hline AOV1 & 9 & 2 & 30 \\
\hline AOV2 & 7 & 9 & 5 \\
\hline AOW1 & 0 & 2 & 0 \\
\hline AOW2 & 2 & 2 & 3 \\
\hline AOW3 & 0 & 0 & 2 \\
\hline AOW5 & 0 & 2 & 1 \\
\hline AOX1 & 5 & 3 & 10 \\
\hline AOX2 & 0 & 2 & 3 \\
\hline AOW $^{a}$ & $27^{b}$ & $27^{b}$ & ND \\
\hline
\end{tabular}

a Cat AOW4 died 140 days post infection with only immature adults and fragments

b Viability not determined ND; not determined

\section{Heartworm antigen testing}

HWAg assays were performed using the PetChek ${ }^{\circledR}$ Canine Heartworm Antigen Test (IDEXX ${ }^{\circledR}$ Laboratories, Westbrook, ME), an enzyme immunoassay for the detection of $D$. immitis antigen.

\section{Heartworm antibody testing}

An indirect microtiter plate ELISA was developed and optimized for research purposes using 2 different heartworm-specific recombinant antigen targets: HWAg-1 (Hong et al. 1996, Frank et al. 1998) and HWAg-2 (Poole et al. 1992). Sample and conjugate diluents contained non-specific proteins and detergent. Test wells coated with either of the antigen targets were incubated with diluted serum sample (1:400). After a wash step, detection of anti-heartworm specific antibody was performed using a secondary anti-species antibody horseradish peroxidase conjugate (1:2000 dilution, Jackson ImmunoResearch, West Grove, PA). Following a wash step and addition of substrate, plate absorbance optical density (OD) values were recorded and sample-to-positive ratios (S/P; sample OD divided by positive control OD) were calculated for each sample (sample OD-negative control OD]/positive control OD-negative control OD) (Buddle et al. 2013). Samples with $\mathrm{S} / \mathrm{P}$ ratios $\geq 0.5$ were positive. The optimal S/P ratio for the test cutoff was chosen by testing known positive and negative samples and was verified using a receiver operator curve (ROC) analysis using JMP software (SAS Institute, Inc., Cary, NC).

\section{Results}

\section{Necropsy results}

At necropsy, adult worms or fragments of dead worm were recovered from 10 of 12 cats; only worm fragments were recovered from a single cat (AOW3). Neither adult worms nor worm fragments were recovered from 2 cats (AOS1 and AOU1). Cat AOW4 succumbed to infection after day 140 p.i. (Table 1). 


\section{A. Results of heartworm antigen assay}
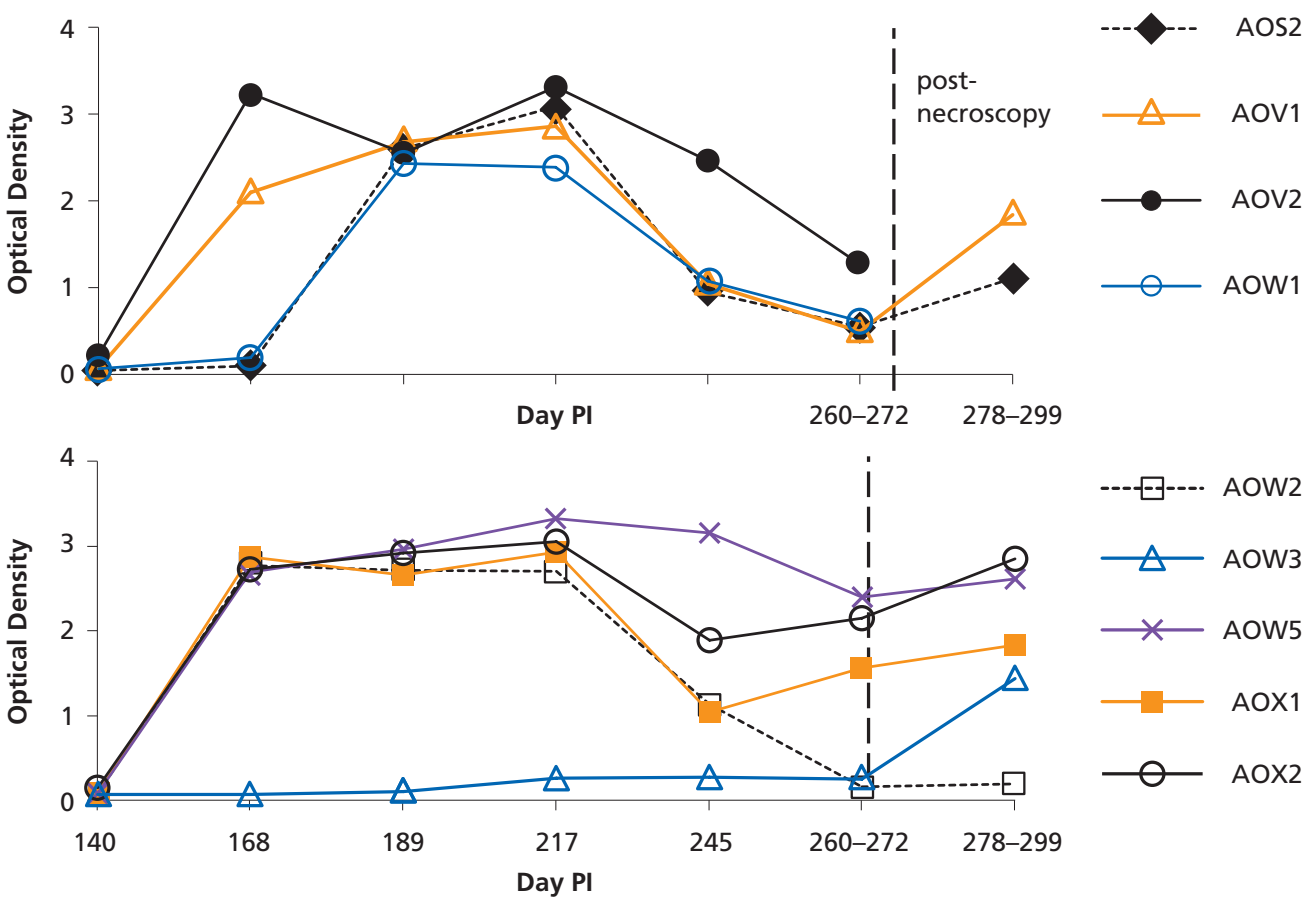

$\triangle-$ AOW3

$\longrightarrow$ AOW5

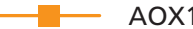

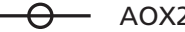

Fig. 1 (A) Results of heartworm antigen assays for temporal samples collected between days 140 to 299 post-infection. Assay cutoff was $0.103-0.135$; samples prior to day 140 were negative. Cat AOW4 succumbed to infection 140 days PI (ELISA OD 0.379 at day 140), post-necropsy samples were not obtained for cats AOV2 and AOW1, cats AOS1 and AOU1 were negative at all time points (S/P ratio $<0.075$, data not shown). Day 299 blood sample was collected from the heart during necropsy.

\section{Antibody and antigen detection assays}

Heartworm-specific antibody was first detected as early as 42-56 days p.i. depending on the antigen target used (Table 2). Eleven of 12 cats were HWAg-1 antibody-positive 68 days p.i.; the remaining cat (AOW2) was positive on day 140 p.i. All but one cat was initially positive between $42-84$ days p.i. in the HWAg-2 antibody assay; the remaining cat (AOW2) was negative at all time points, despite having 4 adult worms recovered at necropsy (Fig. 1).

The 2 cats (AOS1, AOU1) that had no detectable circulating antigen, worms, or fragments developed detectable antibody in both the HWAg-1 and HWAg-2 assays. Both cats were antibody positive in both assays at day 68 days p.i., and subsequently produced assay results that declined over time. Cat AOS1 was negative in the HWAg-1 and -2 assays days 140 and 217, respectively, whereas AOU1 was negative in the HWAg-1 assay day 270, and was weakly positive in the HWAg-2 assay for the duration of the study. No significant decline in antibody concentration was observed in HWAgpositive cats. A single cat (AOW3), which had only worm fragments at necropsy, was reactive in the HWAg-1 and -2 antibody assays at 68 and 84 days p.i., respectively.

Circulating antigen was detected in 10 of the 12 cats in this study; adult worms or fragments were found in each of the antigen-positive cats. Neither adult worms nor worm fragments were observed in the 2 antigen-negative cats (Table 2). In 2 cats (AOW4, AOV1) antigen was detected as early as 140 days p.i., in 6 cats at 168 days p.i., and in the remaining 2 cats at 189 and 217 days p.i. 
B. Results of heartworm antibody assay using heartworm antigen HW Ag-1

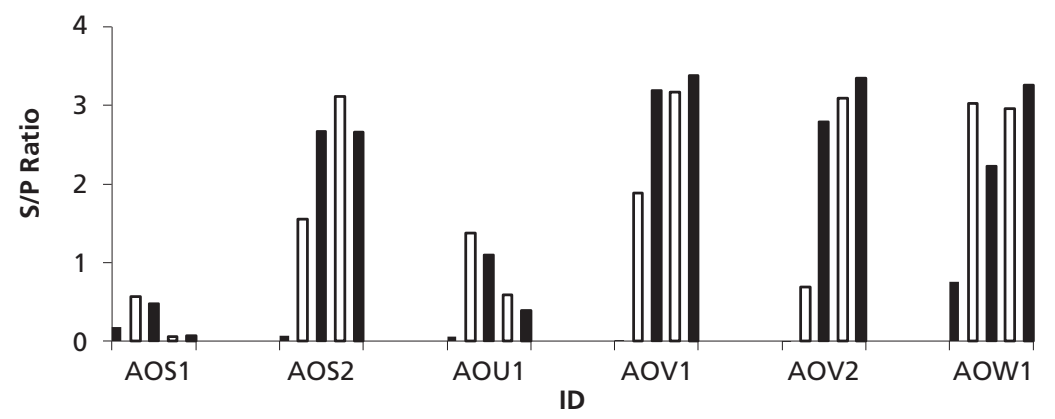

$$
\begin{gathered}
\quad \text { Days } \\
\text { post infection } \\
\square 56 \\
\square 68 \\
\square 140 \\
\square 245 \\
\square 270-299
\end{gathered}
$$

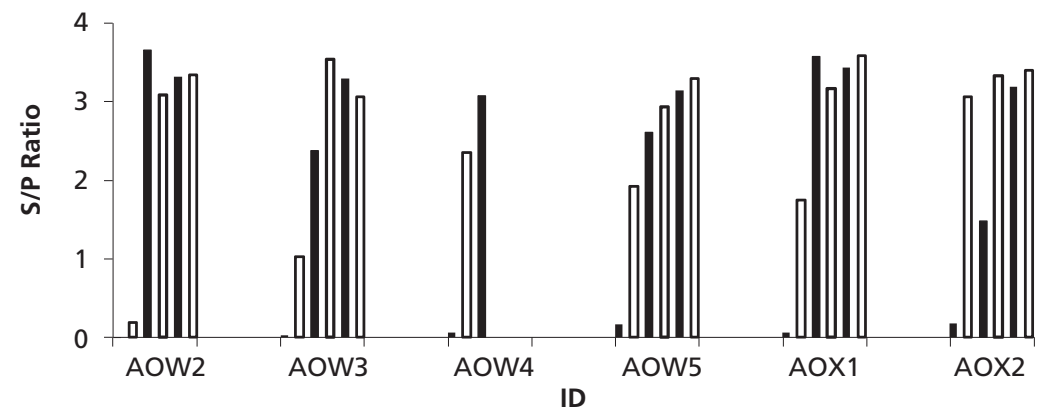

Days

post infection

- 56

$\square 68$

- 140

ㅁ 189

— 245

ㅁ 270-299

C. Results of heartworm antibody assay using heartworm antigen HW Ag-2

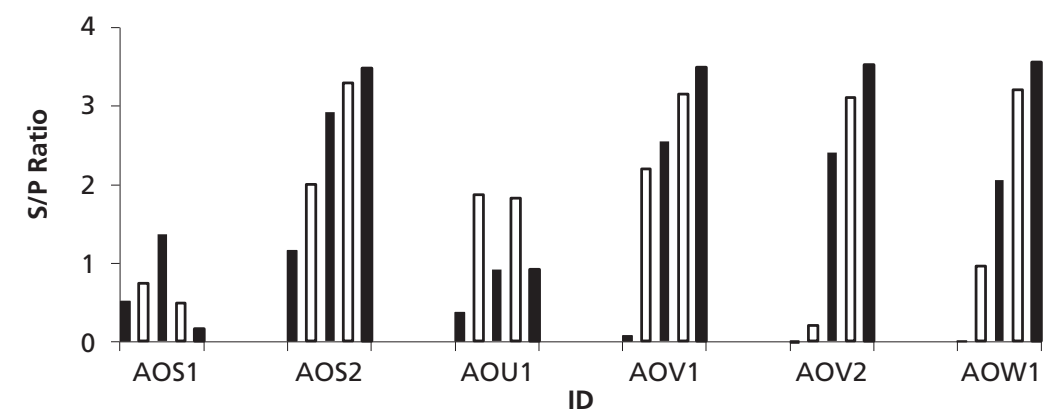

Days

post infection

- 56

$\square 68$

- 140

ㅁ 245

- 270-299

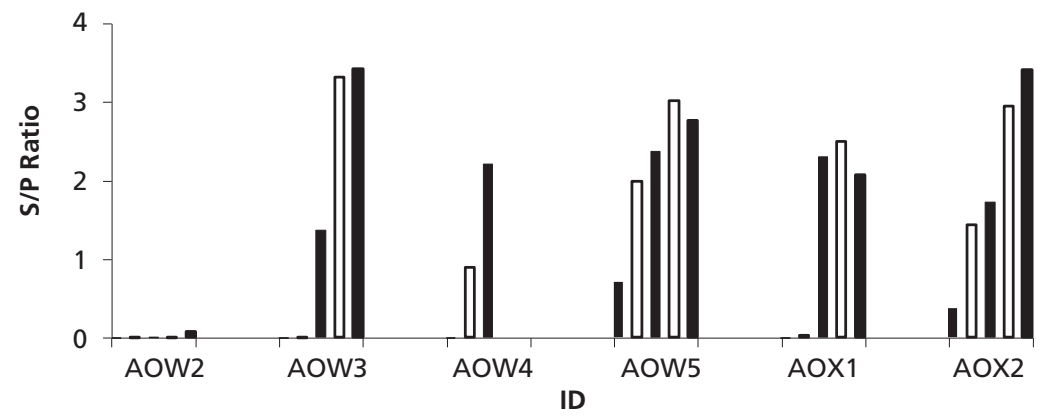

Days

post infection

- 56

ㅁ 68

- 140

ㅁ 245

- 270-299

Fig. 1 (B) and (C) Results of heartworm antibody assay (S/P Ratio) using HWAg-1 (B) and HWAg-2 (C) ELISAs for samples between days 56 and 299 post-infection. Assay cutoff was 0.5; samples collected prior to day 56 were negative. 
Table 2 Days post infection cats initially tested positive for heartworm antibody (HWAg-1 or HWAg-2 assays) and heartworm antigen following SC injection of 100 third-stage Dirofilaria immitis larvae on day 0

\begin{tabular}{|c|c|c|c|}
\hline \multirow{2}{*}{ Cat ID } & \multicolumn{3}{|c|}{ Day of initial positive result (day post infection) } \\
\hline & Anti-HWAg-1 & Anti-HWAg-2 & Heartworm Ag \\
\hline AOS1a & 68 & 68 & NA \\
\hline AOS2 & 68 & 42 & 189 \\
\hline AOU1a & 68 & 68 & NA \\
\hline AOV1 & 68 & 68 & 168 \\
\hline AOV2 & 68 & 75 & 140 \\
\hline AOW1 & 56 & 68 & 168 \\
\hline AOW2 & 140 & NA & 168 \\
\hline AOW3 & 68 & 84 & 217 \\
\hline AOW4 & 68 & 68 & 140 \\
\hline AOW5 & 68 & 42 & 168 \\
\hline AOX1 & 68 & 75 & 168 \\
\hline AOX2 & 68 & 68 & 168 \\
\hline
\end{tabular}

a Cats AOS1 and AOU1 had no adult worms at necropsy

Ag; antigen

HW; heartworm

NA; not available

Day 299 sample was collected from the heart during necropsy, and it is likely that increased antigen levels at this time point may be due to higher antigen levels in the heart as a result of adult worm location.

\section{Discussion}

In the present study, antibody was detected in 11 of 12 cats in both the HWAg-1 and HWAg- 2 assays prior to 84 days p.i., which would be prior to clinical symptoms of HARD. One cat (AOW2) was positive in the HWAg- 1 assay at 140 days p.i. and remained positive through the study but was negative at all time points in the HWAg-2 assay, despite the fact that 4 adult worms and 3 worm fragments were recovered at necropsy and circulating HTWM Ag was detected at 168 days. Depending on the antigen target used, anti-heartworm antibody may not be detectable in all cats with adult heartworms. Test sensitivities of $72.4 \%$ and $62.1 \%$ were reported in a study using 2 commercial antibody detection assays in naturally infected cats with necropsy-confirmed infections (Berdoulay et al. 2004). An antigen test may identify cats that have mature adult infections, but also may have falsenegative results on antibody tests (Snyder et al. 2000). The change in antigen and antibody concentration from peripheral blood collected day 270 and right ventricular blood immediately after euthanasia suggests that post-mortem cardiac blood samples may overstate the predictive positive-test results for clinical applications.

Antibody was detected between days 56 and 68 in the 2 cats (AOS1 and AOU1) in which no worms or fragments were detected at necropsy and for which HWAg testing was negative at all time points. In assays of each of these cats, in contrast to cats harboring adult heartworms, antibody concentration declined at subsequent time points and became negative or weak positive by day 270 p.i. Identification of cats that harbor immature worms with no evidence of adult worms is important because 
they may show HARD-related symptoms and have a higher prevalence of pulmonary arterial lesions compared with heartworm-free cats (Browne et al. 2005).

Heartworm antibody was detected at all time points after 68 days p.i. in both antibody assays in cat AOW3, which had 2 worm fragment and no adult worms. Antigen was detected at all time points, suggesting that worm fragments shed detectable levels of antigen into peripheral blood. Although the antigen has generally been associated with full mature female heartworms, these data suggest that antigen can be associated with immature adults only. Further, the exposure of the immune system to a wide array of heartworm antigen could be expected to produce different antibody responses, some of which may be stage specific as demonstrated by the differences in antibody response to HWAg-1 vs HWAg-2.

All 10 cats that had adult worms or fragments at necropsy were HWAg positive. Two cats were initially positive 140 days p.i., 6 were positive 168 days p.i., and the remaining 2 were initially positive on days 189 and 217 p.i. The OD reading obtained for 6 of the 8 cats that were positive at day 168 were high, which would correlate with high levels of circulating serum antigen (Weil et al. 1985). The detection of relatively high levels of HWAg at 12-40 days prior to the assumed stage of sexual maturation suggests that the diagnostic antigen was produced in some form prior to fecundity or worm fragments were in the process of degeneration. We propose that in this experimental model with infection of a large number of $\mathrm{L}_{3}(100)$, the death of large numbers of immature worms may release adequate concentration of diagnostic antigen in the absence of mature female worms. Although this may lead to detectable antigenemia at time points more closely associated with HARD, similar results were not demonstrated in early detection of antigen in other experimental infections (Dillon 2007, Dillon et al. 2013, Dillon et al. 2014).
The study supports results of previous studies, which indicate that both antigen and antibody tests are needed to increase the sensitivity of either single test alone (Snyder et al. 2000, Berdoulay et al. 2004). Two of 12 cats appear to have had a transient exposure and were reactive only in the antibody assay, and 1 cat with confirmed adult infection was non-reactive in a single antibody assay. The utility of both antigen and antibody testing in suspected cases of lung disease consistent with feline heartworm disease is supported by these data.

\section{Conflict of Interest Statement}

The authors declare that they have no potential and actual conflicts of interest with other persons or organizations within 3 years of initiating the work in this study.

\section{Funding Sources}

This study did not receive any specific grant from funding agencies in the public, commercial, or notfor-profit sectors.

\section{Compliance with ethical standards}

All applicable international and national guidelines for the care and use of animals were followed. All procedures performed in the studies involving animals were in accordance with the ethical standards and were approved by The Institutional Animal Care and Use Committees of University of Georgia and Auburn University where the studies were conducted.

\section{Open Access}

This article is distributed under the terms of the Creative Commons Attribution 4.0 International License (http://creativecommons.org/licenses/ by/4.0/), which permits unrestricted use, distribution, and reproduction in any medium, provided you give appropriate credit to the original author(s) and the source, provide a link to the Creative Commons license, and indicate if changes were made. 


\section{References}

Atkins CE, DeFrancesco TC, Coats JR, Sidley JA, Keene BW (2000) Heartworm infection in cats: 50 cases (1985-1997). J Am Vet Med Assoc 217: 355-358

Berdoulay P, Levy JK, Snyder PS, Pegelow MJ, Hooks JL, Tavares LM, Gibson NM, Salute ME (2004) Comparison of serological tests for the detection of natural heartworm infection in cats. J Am Anim Hosp Assoc 40: 376-384

Blagburn B, Dillon AR (2007) Feline heartworm disease: solving the puzzle. Vet Med 10: 7-14

Browne LE, Carter TD, Levy JK, Snyder PS, Johnson CM (2005) Pulmonary arterial disease in cats seropositive for Dirofilaria immitis but lacking adult heartworms in the heart and lungs. Am J Vet Res 66: 1544-1549

Buddle B M, Wilson T, Luo D, Voges H, Linscott R, Marte, E, Lawrence J, Neill M A (2013) Evaluation of a commercial enzyme-linked immunosorbent assay for the diagnosis of bovine tuberculosis from milk samples from dairy cows. Clin Vaccine Immunol 20: 1812-1816

Dillon R (1984) Feline dirofilariasis. Vet Clinics North Am Small Anim Pract 14: 1185-1199

Dillon AR, Blagburn B, Tillson DM, Brawner W, Spencer J, Welles B, Boothe D (2007) Immature heartworm infection produces pulmonary parenchymal, airway, and vascular disease in cats. J Am Vet Intern Med 21: 608-609

Dillon AR, Warner AE, Brawner W, Hudson J, Tillson M (2008). Activity of pulmonary intravascular macrophages in cats and dogs with and without adult Dirofilaria immitis. Vet Parasitol 158: 171-176

Dillon AR, Tillson W R, Hathcock J, Brawner B, Wooldridge A, Cattley R, Welles B, Barney S, Lee-Fowler T, Botzman L, Sermersheim M, Garbarino R (2013) Lung histopathology, radiography, high-resolution computed tomography, and bronchio-alveolar lavage cytology are altered by Toxocara cati infection in cats and is independent of development of adult intestinal parasites. Vet Parasitol 193: $413-426$

Dillon AR, Tillson DM, Wooldridge A, Cattley R, Hathcock J, Brawner WR, Cole R, Welles B, Christopherson PW, Lee-Fowler T, Bordelon S, Barney S, Sermersheim M, Garbarino R, Wells SZ, Diffie EB, Schachner ER (2014) Effect of pre-cardiac and adult stages of Dirofilaria immitis in pulmonary disease of cats: CBC, bronchial lavage cytology, serology, radiographs, CT images, bronchial reactivity, and histopathology. Vet Parasitol 206: 24-37

Frank GR, Mondesire RR, Brandt KS, Wisnewski N (1998) Antibody to the Dirofilaria immitis aspartyl protease inhibitor homologue is a diagnostic marker for feline heartworm infections. J Parasitol 84: 1231-1236
Hong XQ, Santiago Mejia J, Kumar S, Perler FB, Carlow CK (1996) Cloning and expression of DiT33 from Dirofilaria immitis: a specific and early marker of heartworm infection. Parasitol 112: 331-338

Jones S, Graham W, von Simson C, Stannard R, Carithers D, Payne P, Rehm C, Nelson CT, Smith-Blackmore M, Clyde E, Zaffarano B, Miller M, Atkins C, McCall J, Loenser M, Rumschlag T (2014) Current feline guidelines for the prevention, diagnosis, and management of heartworm (Dirofilaria immitis) infection in cats (revised October 2014). http:// www.heartwormsociety.org. Accessed: 10 May 2017

Lee AC, Atkins CE (2010) Understanding feline heartworm infection: disease, diagnosis, and treatment. Top Companion Anim Med 25: 224-230

Lister A, Atkins C, Atwell R (2008) Acute death in heartworm-infected cats: unraveling the puzzle. Vet Parasitol 158: $196-203$

Maia FCL, McCall JW, Silva VA Jr, Piexoto CA, Supakorndej P, Supakornde, N, Alves LC (2011) . Structural and ultrastructural changes in the lungs of cats Felis catus (Linnaeus, 1758) experimentally infected with $D$. immitis (Leidy, 1856). Vet Parasitol 176: 304-312

McCall JW, Dzimiansky MT, McTier TL, Jernigan AD (1992) Biology of experimental heartworm infection in cats. In: Soll, M.D. (ed), Proc 1992 Heartworm Symposium. American Heartworm Society, Batavia, Il, pp 71-79

Poole CB, Grandea AG 3rd, Maina CV, Jenkins RE, Selkirk ME, McReynolds LA (1992) Cloning of a cuticular antigen that contains multiple tandem repeats from the filarial parasite Dirofilaria immitis. Proc Nat Acad Sci 89: $5986-5990$

Snyder PS, Levy JK, Salute ME, Gorman SP, Kubilis PS, Smail PW, George LL (2000) Performance of serologic tests used to detect heartworm infection in cats. J Am Vet Med Assoc 216: 693-700

Weil GJ, Malone MS, Powers KG, Blair LS (1985). Monoclonal antibodies to parasite antigens found in the serum of Dirofilaria immitis-infected dogs. J Immunol 134: $1185-1191$

Weil GJ (1987) Dirofilaria immitis: identification and partial characterization of parasite antigens in the serum of infected dogs. Exp Parasitol 64: 244-251 